\title{
Canine Rabies Outbreaks, Vaccination Coverage, and Transmission in Humans: Greater Accra Region, Ghana- A Retrospective Study-2006-2011
}

\author{
Perdita Hilary Lopes ${ }^{1,}$, , Patricia Akweongo², Fred Wurapa ${ }^{2}$, Edwin Afari ${ }^{2}$, Samuel Oko Sackey ${ }^{2}$, \\ Edward Mark Hansen ${ }^{3}$, Kofi Mensah Nyarko ${ }^{2}$ \\ ${ }^{1}$ Department of Epidemiology, Veterinary Services Directorate, Accra, Ghana \\ ${ }^{2}$ Department of Epidemiology and Disease Control, School of Public Health University of Ghana, Accra, Ghana \\ ${ }^{3}$ Department of Field Services, Veterinary Services Directorate, Accra, Ghana
}

Email address:

pehilopes@yahoo.com(P.H. Lopes),akweongo@gmail.com(P.Akweongo),wurapaf@yahoo.co.uk(F.Wurapa), afariea@yahoo.co.uk(E.Afari), sackey492003@yahoo.co.uk(S. O. Sackey),Dr.e.markhansen@gmail.com(E. M. Hansen), konyarko@yahoo.co.uk(K. M. Nyarko)

${ }^{*}$ Corresponding author

\section{To cite this article:}

Perdita Hilary Lopes, Patricia Akweongo, Fred Wurapa, Edwin Afari, Samuel Oko Sackey, Edward Mark Hansen, Kofi Mensah Nyarko. Canine Rabies Outbreaks, Vaccination Coverage, and Transmission in Humans: Greater Accra Region, Ghana- A Retrospective Study-20062011. American Journal of Clinical and Experimental Medicine. Vol. 6, No. 2, 2018, pp. 58-63. doi: 10.11648/j.ajcem.20180602.14

Received: April 10, 2018; Accepted: April 26, 2018; Published: May 21, 2018

\begin{abstract}
Rabies is a highly fatal, viral, zoonotic disease caused by a Lyssa virus. It is acquired through the bite of an infected animal, mostly dogs. Estimated annual global human mortalities from rabies is 61,000, over 99\% of which are from developing countries where about US\$583.5 million is spent on its control. Rabies is enzootic in Ghana. Vaccinating about $70 \%$ of the dog population leads to a reduction in rabies transmission to humans; however, percentage dog anti-rabies vaccination coverage in Ghana is unknown. This study therefore investigated the magnitude of rabies in dogs and humans in the Greater-Accra region, and assessed annual percentage dog anti-rabies vaccination coverage from 2007-2011. Secondary data on rabies vaccination, post mortem and dog quarantine records for all ten districts in the region, and human rabies records were analysed. Means and percentages were calculated, graphs drawn and trends analysed. The number of animal samples which tested positive out of 309 , was 283 . Predictive value positive was $91.6 \%$. Of the positive cases, 97.5\% (276/283) had no previous vaccination history, and 96.1\% (272/283) were from dogs. Fifty-five out of 174 (31.6\%) samples were from dogs which bit more than one person. Human exposures to dog and other animal bites are not differentiated. Average annual number of outbreaks was 31, whereas percentage dog vaccination ranged from 10.26-17.56. Current annual percentage dog anti-rabies vaccination coverage is very low, whereas the incidence of dog rabies in the region is high. The number of humans affected may be underestimated. Government should immediately facilitate annual mass vaccination of pets.
\end{abstract}

Keywords: Rabies, Lyssa Virus, Dogs, Percentage Vaccination Coverage, Greater-Accra Region, Ghana

\section{Introduction}

Rabies is a highly fatal, viral disease with an insidious onset, caused by a Lyssa virus of the family Rhadoviridae. It is a multi-species, zoonotic disease which affects mainly warm-blooded animals [1-3]. Infection usually follows the bite of a rabid animal [1,3].The virus is neurotropic, travelling by peripheral nerves through the central nervous system, and finally ending up in the brain where it causes encephalopathy [1-4]. Rabies occurs in two forms; furious and dumb rabies [1, 4-6]. The former is easier to diagnose clinically based on symptoms. However, in dumb rabies, due to the absence of characteristic aggressive symptoms often associated with the disease, diagnosis may be missed, [1, 46], making the disease even more dangerous. Moreover, once symptoms of central nervous system involvement manifest, rabies is nearly always $100 \%$ fatal $[1,4,7]$. It has been 
established that over $90 \%$ of human rabies exposures are from dogs, and of these, $99 \%$ are from free-roaming dogs. Additionally, 99\% human rabies deaths are from rabid dogs [7]. All these facts therefore, suggest that control of rabies in humans is hinged on its control in dogs. This has been adequately proven by examples from a number of countries which have eliminated canine rabies through control of the disease in dogs $[10,11]$. In yet some other countries, significant strides have also been made in canine rabies reduction through vaccination, with a concomitant reduction in human rabies. Despite all these available evidence, rabies caused an estimated 61,000 human deaths globally in the year 2010, with over $95 \%$ of these occurring in Asia and Africa alone [11, 13]. A more recent study put the annual global estimates of human rabies deaths at 59,000. In Ghana, 25 cases of human rabies were reported from 2009-2011. From an economic perspective, between US\$6-8.6 billion is estimated to be spent on rabies control globally each year $[11,14]$, whereas other studies suggest that about US $\$ 124.2$ billion could be saved annually with canine rabies control through dog vaccination. In Africa and Asia, an estimated US\$583.5 million is spent on rabies control annually. This is in sharp contrast to developed countries such as the United States, where an estimated $\$ 300$ million is spent annually on rabies control $[7,8]$. In the later, though, the greater part of this expenditure is concentrated on dog vaccinations [7, 8]. Furthermore, high stray dog populations in developing countries means that a single stray, rabid dog becomes a source of infection for several others as they mingle freely with each other [17, 18]. This poses high risks of rabies infection to humans if such uncontrolled gatherings of dogs are unvaccinated or have low percentage vaccination coverage. As in any infectious disease, adequate herd effect is required to break the cycle of transmission [20,21]. Different studies have different postulates on the percentage vaccination coverage (PVC) which would be adequate to achieve this with respect to rabies. A study has shown that, when a percentage vaccination coverage (PVC) of 70 of the dog population was achieved in two successive years, followed by at least a PVC of 50 for 5 consecutive years, it was possible to break the canine cycle of rabies transmission to humans [22]. The World Health Organization (WHO), also recommends a PVC of at least 70 in rabies endemic areas. Another study, however, suggests that canine PVC of 39-57 is adequate to break the cycle of transmission. Finally, global health partners such as the WHO, World Animal Health Organization (OIE), the Global Alliance for Rabies Control (GARC) all agree that elimination of dogmediated human rabies is possible, since all the tools needed for this are available. Clearly then, human rabies elimination is inextricably linked to elimination of the disease in dogs. Canine and human rabies have thus been targeted for elimination by the year 2030, and Ghana, being a part of the international community, must work towards this. With this background, an analysis of rabies data in the Greater-Accra Region (GAR) of Ghana was carried out to determine the magnitude of animal rabies from 2007-2011, to establish the trend of rabies outbreaks in animals and humans, and to assess the percentage canine anti rabies vaccination coverage for the same period.

\section{Materials and Methods}

\subsection{Study Area and Study Population}

The study area was the GAR of Ghana, which is located in the south-east of the country along the Gulf of Guinea, between latitude $5^{\circ} 33^{\prime} 0^{\prime \prime}$ North, $0^{\circ} 13^{\prime} 0^{\prime \prime}$ West. It occupies a land surface of 3,245 square kilometers, and an estimated population of $4,010,054$. The region is divided into ten administrative and political districts. A survey of the dog population during polio vaccination exercises carried out by the Ministry of Health in 2009 estimated the number of dogs in GAR at 82,684 .

\subsubsection{Study Type and Sources of Data}

Secondary data on official monthly veterinary records on rabies incidence in GAR for the period 2007-2011, were reviewed. These comprised data on dogs quarantined for suspected rabies, rabies tests carried out at the Accra Veterinary Laboratory, and monthly reports from the ten districts in the GAR. Human rabies morbidity and mortality data from 2008-2011 from the Public Health Division of the Ghana Health Services, GAR, was also reviewed.

\subsubsection{Data Analysis}

Data was analysed and the results expressed as means, frequencies, and percentages, and the trend in animal vaccinations over the period determined. The number of human involvement with suspected rabid dogs were also analyzed.

\subsection{Ethical Approval}

Permission was sought and obtained from the Director of Veterinary Services and the School of Public Health, University of Ghana, Legon, prior to the study.

\section{Results}

\subsection{Number of Suspected Samples Submitted for Rabies Testing and Animal Species Involved}

A total of 319 samples from different animal species were submitted to the Accra Veterinary Laboratory on suspicion of rabies from 2007-2011. The highest number of samples submitted was from dogs; $95.6 \%(305 / 319)$, contributions from other species were cats and horses, $2.5 \%$ (8/319), $0.9 \%(3 / 319)$ respectively. The rest were $1 \%$ for goat, pig and rabbit (1/319) each. Out of the 319 samples submitted, 3.1\% (10/319) were decomposed so were not tested. Out of the number of samples tested for rabies, 283/309 were positive; predictive value positive (PVP) was $91.6 \%$. The proportion of dog samples out of the total number of positives was $96.1 \%$ (272/283). During the period of review, a total of 1,146 dogs were quarantined on suspicion of rabies, with the highest and lowest numbers, 182, and 254 being in 2009 and 2008 respectively. 


\subsection{Rabies Test Result and Vaccination Status of Animas from Which Samples Were Submitted}

Table 1. Vaccination status of animals from which samples were submitted for rabies testing.

\begin{tabular}{llll}
\hline Vaccination status & Positive & Negative & Total \\
\hline Vaccinated & 7 & 0 & 7 \\
Unvaccinated & 276 & 26 & 302 \\
Total & 283 & 26 & 309 \\
\hline
\end{tabular}

An analysis of the vaccination status of animals from which samples were submitted for rabies testing is shown in Table 1. Of the 283 samples that tested positive for rabies out of $309,97.5 \%(276 / 283)$ were unvaccinated. All seven samples, which came from vaccinated dogs, however, also tested positive for rabies.

\subsection{Number of Rabies Outbreaks and Percentage Vaccination Coverage in GAR, 2007-2011}

From 2007-2011, out of an estimated 82,684 dogs in the GAR, the highest annual number of dogs vaccinated was $14,521(17.6 \%)$. The corresponding annual PVC for dogs over the peroid of review, as well as the number of reported rabies outbreaks are shown in Figure 1. The lowest and highest PVC, 10.26 and 17.56, were in 2008 and 2011 respectively, whereas the number of outbreaks ranged from 19-55, the lowest and highest being in 2007 and 2011 respectively.

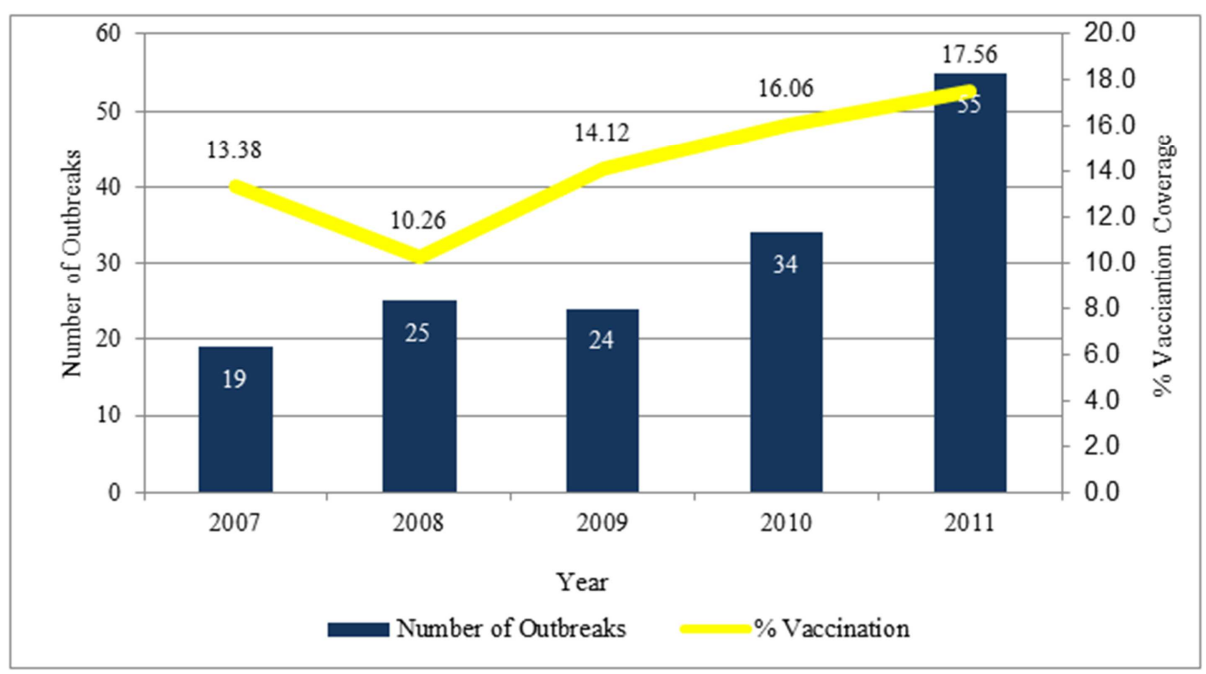

Figure 1. Annual rabies outbreak and percentage dog vaccination coverage, Greater-Accra Region, 2007-2011.

Dog vaccinations were mostly carried out based on individual requests by owners, however, there were also mass vaccinations carried out in $6 / 10$ districts. Mass vaccinations were not syynchronised across the region, but rather, each district carried out its own schedule of vaccinations. Additionally, they spanned several weeks, sometimes months, and were limited in scope, as shown by the PVC. All vaccinations were on owner-pay principles, with some limited support from the local authorities.

\subsection{Human Involvement with Rabies Suspected Dogs for Which Samples Were Submitted for Rabies Test}

Of the samples submitted to the laboratory for rabies test, 174/319 was from dogs which bit 324 humans; of these, $55 / 174(31.6 \%)$ bit more than one person. Further, twenty percent $(64 / 324)$ of victims were bitten by their own dogs.

\subsection{Human Morbidity and Mortality Data from Rabies}

From the Public Health Division of the Ghana Health Service, GAR, a total of 15,896 reported animal bite cases involving humans were recorded for the period under review. However, there was no data on what proportion of this figure was from potentially rabid dogs as all animal bite cases were lumped together. There was also, no available data on the number of humans who were given post exposure vaccination for rabies; animal bite victims had their wounds sanitized, and were given anti-tetanus injection and antibiotic cover.

\section{Discussions}

The highest annual PVC for dogs for the period was 17.56, in 2011. One of the reasons for the low PVC could possibly be due to "owner-pay policy", resulting in low patronage. This was consistent with findings from a number of studies on dog vaccinations in developing countries [9, 28-29]. In contrast, when canine vaccinations were free, patronage was higher, and consequently, higher vaccination coverage was achieved [9, 28, 29, 30]. Interestingly, the highest number of rabies outbreaks in GAR was also recorded in the same year, 2011 when the highest PVC was achieved. This was contrary to the notion that increasing PVC resulted in an automatic decrease in rabies incidence. This can be explained by the fact that even the highest PVC of 17.5 was still inadequate to break the transmission cycle as it was clearly, far below even the minimum recommendation of 37.5. Another study also reported that even though a PVC of 50 could break the transmission cycle, it could not lead to rabies elimination in 
dogs. However, a PVC of 70 was sufficient to interrupt human exposure to the virus. All these studies agree that at least, a certain minimum percentage of dogs should be vaccinated in a given geographical location within a defined period in order to break the canine transmission cycle of rabies.

The rather high number of samples which tested positive to rabies, but had no previous vaccination history, as well as the high number of outbreaks are further confirmation of the finding of low PVC in the region. Additionally, the high PVP points not only to the high sensitivity of the test, but also, high prevalence of rabies in the dog population. Of particular concern is the finding that all seven samples from animals which were vaccinated within the year prior to the test were positive for rabies. This could probably be due to vaccine failure which could be attributed to several reasons, such as vaccination by quacks who use fake vaccines, or a break in the cold chain cycle. This finding is consistent with studies from both the developed and developing countries which have also reported rabies in dogs previously vaccinated against the disease [32-35].

The study shows that the majority of positive samples were from dogs. Furthermore, over, $98 \%$ of the reported human involvement with suspected rabid animals from which samples were tested, was also from dogs as found in similar studies [31, 36, 37]. Both of these observations support the World Health Organization's assertion that the majority of human exposures to rabies are from rabid dogs $[8,10,12]$.

Since human exposures to dog bite cases were lumped together with other animal bite cases at the Public Health Unit, Ghana Health Service, GAR, it was not possible to glean exactly how many humans had had potential exposures to rabid dogs, nor whether they were followed up. Furthermore, it was also not clear whether the 324 dog bite victims recorded by the Accra Veterinary Laboratory were part of this figure. From literature, about $80 \%-90 \%$ animal bite cases involving humans are from dogs. This means out of the 15,896 animal bite cases reported at the Public Health Division of the Ghana Health Service, GAR, at least 12,717 could have been from dogs. This can be compared to studies in Asia where, out of 3,529,000 animal bite cases, 31,539 human rabies deaths were reported. Similarly in Africa, out of 802,000 animal bite cases, 23,823 human deaths were recorded [17,39]. Further, considering that rabies is endemic in Ghana and GAR for that matter, it is possible that some of the animal bite cases were from potentially rabid dogs. Hence there is the need for animal bite cases to be treated as potentially rabid cases and thoroughly investigated. In furtherance to this, all dog bite cases should be identified and reported as such by medical practitioners. In some jurisdictions of the developed nations, all animal bite cases are referred to the local veterinary authority who apprehend the offending animal, quarantine and observe it for at least 10 days. This is to ensure that the offending animal is not potentially rabid. Based on other epidemiological information, the exposed human is then given the post- exposure prophylaxis. However, it is not clear from available data how many of the dogs quarantined at the Veterinary Services Directorate, were referrals from human health hospitals, nor how many were self- reported. This would have helped to link the human victims to all animal rabies suspected cases.

\section{Limitations}

The dog population used was only from data captured from homes with children under five years of age during the polio immunization exercise. Additionally, stray dogs could have been missed in the count. This means that the actual dog population could be far in excess of what was used, which would have affected the PVC obtained. Further, the same population was used as the denominator for the analysis over the five year period. Since populations are not static, this could also have affected PVC obtained. Additionally, rabies cases captured reflect only those that were reported to the VSD.

We did not take into consideration other issues such as vaccination strategy, that is; central point as against door to door vaccination, or resource availability as a contributing factor to PVC.

\section{Conclusions}

The prevalence of rabies in the region is very high. On the other hand, the PVC is so low that it is not making any dent in the transmission cycle of rabies. A lot of potential human rabies cases are also not being captured by the Ghana Health Service. The Ministry of Food and Agriculture, through the Veterinary Services Directorate, should immediately facilitate mass vaccination campaign for pets in order to encourage participation and target a vaccination coverage of $70 \%$ or more. This should be continued annually for at least five consecutive years as recommended by the WHO. Additionally, the Public Health Department of the Ghana Health Service and the Veterinary Services Directorate should strengthen their collaboration with each other so as to improve rabies surveillance. This will ensure that potential human rabies cases are quickly intercepted.

\section{Acknowledgements}

The authors wish to express their sincere appreciation to the Director of Veterinary Services, Dr. Edward Mark-Hansen for granting us permission to carry out this study. We also thank the Head of the Accra Veterinary Laboratory, Dr. Joseph Awuni and his staff for their cooperation in making rabies test results available to us. Special thanks to Dr. Nathaniel Yebuah for giving us access to rabies data, Mr. Joseph Mends of the Accra Metropolitan Rabies Desk, Greater Accra Regional Health Directorate for making available to us animal bite cases for the region, and Dr. Ernest Kenu for his assistance in accessing human rabies records from the Korle-Bu Teaching Hospital. We also thank the Greater Accra Regional Public Health Directorate for sharing data on animal bite cases. Finally, much appreciation goes to all faculty and administrative staff of the Ghana Field 
Epidemiology and Laboratory Training Programme, School of Public Health, University of Ghana, Legon, for their invaluable support.

\section{References}

[1] Overview of rabies. The Merck Veterinary Manual. www.merckmanuals.com/vet/nervous system/rabies/overview _of_rabies.html

[2] W. Drew, Rabies. In Ryan KJ, Ray CG. Sherris Medical Microbiology, 3rd ed., Mcgraw-Hill Medical, 2004, pp 597600 .

[3] Ruprecht Charles. Rhabdoviruses: Rabies virus. In Medical Microbiology. http://www.ncbi/nlm.nih.gov/books/NBK8618

[4] Hemachudha, T., J. Laothamatas, C. E. Rupprecht (2002). Human rabies: a disease of complex neuropathogenetic mechanisms and diagnostic challenges. Lancet Neurology: 1(2) pp. 101-109.

[5] Fekadu M. Canine rabies (1993). Onderstepoort J Vet Res. 60(4):421-7.

[6] Hemachudha T., Mitrabhaki E. Rabies In Davis LE, Kennedy PGE. Infectious Diseases of the Nervous System. Oxford, Butterworth-Heineman, 2000, pp 401-444.

[7] CDC - Rabies around the World - Rabies [Internet]. 2011 [cited 2014 Sep 29].

http://www.cdc.gov/rabies/location/world/index.html?s_cid=c s 521

[8] World Health Organization. WHO Expert Consultation on Rabies. First Report. 2004. WHO Technical Report Series 931. Geneva, Switzerland.

[9] Cleaveland, S., M. Kaare, D. Knobel, M. K. Laurenson (2006). Canine vaccination-providing broader benefits for disease prevention. Vet Microbiology: 117(1)43-50.

[10] Bourly H., L. Dacheux, C. Strady, A. Mailles. Rabies in Europe in 2005 (2005). Euro Surveillance 10: 213-216.

[11] World Health Organization. "WHO Expert Consultation on Rabies. Second report, World Health Organization Technical Report Series 982, 2013; 7-10.

[12] Schneider M. C., A. Belotto, M. P. Ade, S. Hendrickx, L. F. Leanses (2007). Current status of human rabies transmitted by dogs in Latin America. Cadernos de Saude Publica; 23:20492063.

[13] World Health Organization Factsheet 99, 2014. www.who.int/mediacentre/factsheets

[14] Hampson K., L. Coudeville, T. Lembo, M. Sambo, A. Kieffer, et al. (2015). Estimating the global burden of endemic canine rabies. (2015). PLOS Neglected Tropical Diseases.9(5)e0003786 htpps://doi.org/10.1371/journal

[15] Veterinary Public Health Officer GAR. Human Rabies Deaths in Greater Accra, 2007-2011. 2011.

[16] Anderson A., S. A. Swiff, (2013). The cost of canine rabies on four continents. Transboundary and Emerging Diseases; doi:10.1111/tbed.12168.
[17] D. Knobel, (2005). Re-evaluating the burden of rabies in Africa and Asia. Bulletin World Health Organization, 83(5)361.

[18] Jackman J, and A. Rowan (2007). Free-roaming dogs in developing countries: the benefits of capture, neuter and return programs. Chapter 3. The State of the Animals IV. 5577.www.fao.org/fileadmin/user_upload/animalwelfare/1_CNV R\%20Jackman\%20and\%0Rowan\%20(2).pdf

[19] Reece, J. F. Dogs and dog control in developing countries. In DJ Salem and AN Rowan (eds). The State of the Animals III. 2005 66-64. Washington, DC: Humane Society Press.

[20] John, T. J., R. Samuel (2000). Herd immunty: a rough guide. European Journal of Epidemiology; 16(7)601-606.

[21] Fine P., K. Eames, D. L. Heyman (2011). "Herd immunity"-a rough guide. Clinical Infectious Diseases: 52(7)911-916.

[22] Bögel K, F. X. Meslin (1990). Economics of human and canine rabies elimination: guidelines for programme orientation. Bulletin World Health Organization: 68(3):281-91.

[23] Coleman P and C. Dye (1996). Immunization coverage required to prevent outbreaks of dog rabies. Vaccine: 14 (3):185-186.

[24] World Health Organization. Organization des Epizooties. Rationale for investing in the elimination of dog-mediated human rabies. Report of the Global Rabies Conference, Geneva, Switzerland. Available at $<$ http://apps.who.int/iris/bitstream/10665/204621/1 WHO_HTM_NTD_NZD_2016.02.eng.pdf $>$

[25] Ghana Statistical Service. 2010 Population and Housing Census. Final Results. Ghana Statistical Service; 2012.

[26] Ghana Health Service. Head Office, Accra. Dog and Cat Census, Ghana, 2009: Ghana Health Service.

[27] Ghana Health Service, Greater Accra Regional Branch. Animal Bite Cases Adabraka Polyclinic: Ghana Health Service, Greater Accra Regional Directorate; Adabraka, Accra.

[28] Kaare M., T. Lembo, K. Hampson, E. Ernest (2009). Rabies control in rural Africa-evaluating strategies for effective domestic dog vaccination. Vaccine: 27(1)152-160.

[29] Kitala P. M., J. J. McDermott, P. G. Coleman, C. Dye (2002). Comparison of vaccination strategies for the control of dog rabies in Machakos District, Kenya. Epidemiology and Infection 129(1)215-222.

[30] Jibat, T., Hogeveen, H., Mourits, M. C (2015). Review of dog rabies vaccination coverage in Africa, a question of dog accessibility or cost recovery. PLoS Neglected tropical Diseases, 9(2) e0003447.

[31] Zinsstag J., S. Durr, M. A. Penny, R. Mindekem, F. Roth, S. Gonzalez Mendelez Gonzalez, S. Naissenger, J. Hattendorf (2009). Transmission dynamics and economics of rabies control in dogs and humans. Proceedings of the National Academy of Sciences of the United States of America: 106(35)14996-15001.

[32] Oboegbulem S. I., M. I. O Okolo, E. E. Erojikwe (1987). Rabies in vaccinated dogs: observations in eastern Nigeria. Revue scientifique technique. Office International Des Epizooties: 6(1)69-76. 
[33] Blancou J., J. P Firon, P. E. Rollin (1983). Absence of immunity response in dog after vaccination against rabies. Rec. Med. vet. 159(10)789-793.

[34] Kappus K. D. (1976). Canine rabies in the United States 19711973. American Journal of Epidemiology: 103 (3)242-249.

[35] Hadad N., J. Blancou, A. Gritli, A. M. Koutchouk (1985). Study of the activity of two rabies vaccines in Tunisian dogs. Maghreb Vet 2(8) 13-16.

[36] Sudarshan M. K., S. N. Madhusudana, B. J. Mahendra (2007). Assessing the burden of human rabies in India: results of a national multi-center epidemiological survey. International Journal of Infectious Diseases: 11(1):29-35.

[37] Mazigo H. D., F. O. Kumu, E. J. Kweka (2010). Retrospective analysis of suspected rabies cases reported at Bugando Refferral Hospital, Mwanza, Tanzania. Journal of Global Infectious Diseases: 2(3), 216-220.

[38] Massani R., L. Masini (2006). Relationships among injuries treated in an emergency department that are caused by different kinds of animals: epidemiological features. European Journal of Emergency Medicine: 13(3), 160-164.

[39] National Institute of Allergy and Infectious Disease. Rabies Deaths and PEP. Available at: $<$ https://www.niaid.nih.gov/news/events/meetings/Viral\%20 infections/Documents/wilde.pdf $>2005$

[40] Manning S. E., F. D. Ruprecht, C. A. Hanlon (2008). Human Rabies Prevention- United States, Recommendations and Reports MMWR 2008; 57 (RR03); 1-26, 28. 